\title{
Scattering on a chain of zero-range potentials with internal structure in the stochastic magnetic field
}

\author{
A. E. Ryzhkov \\ ITMO University, Kronverkskiy, 49, St. Petersburg, 197101, Russia \\ verdad60@mail.ru
}

PACS 02.30.Tb

DOI 10.17586/2220-8054-2018-9-6-703-710

\begin{abstract}
Scattering problem on an infinite chain of zero-range potentials with internal structure in the stochastic magnetic field is investigated. Model operator is constructed using the perturbation theory for the self-adjoint operators. The relations with the scattering problem without stochasticity are investigated.
\end{abstract}

Keywords: operator extension, stochastic magnetic field, scattering problem.

Received: 1 October 2018

Revised: 20 November 2018

\section{Introduction}

Nanostructures having the form of chains of short-range potentials are widely used in nanotechnologies. Berezin and Faddeev (see [1]) showed that the Hamiltonian with zero-range Fermi type potential is just an extension of a suitable defined symmetric operator. Later, it has been shown by Pavlov [2], that the structure of the standard point interaction models can be enriched substantially when the self-adjoint extensions are constructed in a wider Hilbert space. This idea yields various models of zero-range interaction with an additional internal structure (see, e.g., [3-5]). In papers [6,7] this method has been used to construct and investigate explicitly solvable models of the scattering of the neutron on a point nucleus, whose internal structure depends on a stochastic magnetic field, and of the scattering of acoustic waves on a stochastic point defect with an internal structure.

In the present paper, an exactly solvable model of the neutron scattering on the one-dimensional infinite chain embedded into the three dimensional configuration space $\mathbb{R}^{3}$. We will suppose that the chain is inserted into the stochastic magnetic field. The nuclei in the chain are assumed to be equivalent with the internal structure dependent on the magnetic field. This model corresponds to the case, when the whole chain belongs to one magnetic domain. In the absence of the stochasticity, such model was investigated by Albeverio, Gesztesy, Hoegh-Krohn, Holden [8], Karpeshina [9], Subramanian [10], Kurasov and Pavlov [11]. It was shown that the spectrum of the related operator is purely continuous and consists of two branches:

1) Scattered waves branch $\sigma_{s}$; corresponding eigenfunctions are defined by free waves reflected by the lattice. This branch coincides with the spectrum of the free Laplacian $-\Delta$ in $L_{2}\left(\mathbb{R}^{3}\right)$.

2) Waveguide branch $\sigma_{w}$; corresponding eigenfunctions are localized in a vicinity of the lattice. In the discussing periodic case these functions are of the Bloch type.

It will be shown that the spectral properties of the problem with the stochastic filed are related to the properties of the problems without any stochasticity.

\section{Model operator}

This section is devoted to the construction of the model operator describing scattering in the stochastic magnetic field. Let $L_{2}\left(\mathbb{R}^{3}\right)$ be an external space and free Laplacian $-\Delta$ defined on $W_{2}^{2}\left(\mathbb{R}^{3}\right)$ be an unperturbed operator, simulating the Hamiltonian of the free neutron. Let $E^{i n t}=\oplus \Sigma_{n} E_{n}$ be an orthogonal sum of unitary equivalent finite-dimensional Hilbert spaces. We will restrict our consideration to the simplest case, $E_{n}=\mathbb{C}^{2}, n \in \mathbb{Z}$. Let $A_{n}$ be selfadjoint operators in $E_{n}$ which are mutually unitary equivalent. We will consider, as in the paper [6]:

$$
A_{n}=A(H(\tau)) \equiv \operatorname{diag}\left\{\lambda_{0}, \lambda_{1}\right\}+\sigma_{3} H(\tau), H(\tau)= \pm e_{z},
$$

Where $\lambda_{0}, \lambda_{1}$ are the "levels" of the nucleus, $\sigma_{3}=\left(\begin{array}{ll}q & 0 \\ 0 & -q\end{array}\right)$ is Pauli matrix corresponding to the direction of the stochastic magnetic field $H(\tau)$ parallel to the z-axis. Let $A^{\text {int }}=\oplus \Sigma_{n} A_{n}$ then the nonperturbed operator is defined as a direct sum $\mathcal{L}=(-\Delta) \oplus A^{\text {int }}$ in the space $L_{2}\left(\mathbb{R}^{3}\right) \oplus E^{\text {int }}$ of the kinetic energy operator $(-\Delta)$ 
and the "inner" operator $A^{\text {int }}$. The restriction $-\Delta \Rightarrow-\Delta_{0}$ on the linear set $D_{0}^{e x t}$ of all $W_{2}^{2}$-smooth functions in $\mathbb{R}^{3} \backslash\left\{x_{n}\right\}_{n \in \mathbb{Z}}$ which have the following asymptotic:

creates the boundary form:

$$
u(x) \underset{x \rightarrow x_{n}}{\sim} \frac{u^{n_{-}}}{4 \pi\left|x-x_{n}\right|}+u^{n_{0}}+o(1),
$$

$$
J_{e x t}(u, v)=\left\langle-\Delta_{0}^{*} u, v\right\rangle-\left\langle u,-\Delta_{0}^{*} v\right\rangle=-\sum_{n \in \mathbb{Z}}\left(u^{n_{-}} \overline{v^{n_{0}}}-u^{n_{0}} \overline{v^{n_{-}}}\right) .
$$

Here, $x_{n}=n \vec{e}$ are the positions of nuclei of the lattice.

The restriction of the inner operator $A^{\text {int }} \rightarrow A_{0}^{\text {int }}$ to the linear set $D_{0}^{\text {int }}$ described in $[2,6]$ also leads to nontrivial boundary form:

$$
J_{i n t}(\eta, \xi)=-\sum_{n \in \mathbb{Z}}\left(\eta^{n_{-}} \overline{\xi^{n_{0}}}-\eta^{n_{0}} \overline{\xi^{n_{-}}}\right),
$$

where $\eta, \xi \in E^{i n t}$. We consider here the infinite vectors $\left\{u^{n_{-}}\right\},\left\{u^{n_{0}}\right\},\left\{v^{n_{-}}\right\},\left\{u^{n_{0}}\right\}$, etc., to be elements from $\ell^{2}$. It is obvious that the restricted operators $-\Delta_{0}$ and $A_{0}^{\text {int }}$ have infinite deficiency indices $(\infty, \infty)$. The boundary form $J_{\text {ext }}(u, v)+J_{\text {int }}(\eta, \xi)$ vanishes on the Lagrange planes given by the translation-invariant boundary conditions described in [11]:

$$
\left(\begin{array}{l}
u^{n_{-}} \\
\eta^{n_{-}}
\end{array}\right)=\sum_{m \in \mathbb{Z}} \boldsymbol{\Gamma}_{n-m}\left(\begin{array}{c}
u^{n_{0}} \\
\eta^{n_{0}}
\end{array}\right), \quad \boldsymbol{\Gamma}_{-n}=\boldsymbol{\Gamma}_{n}^{*}, \quad|n|>N \rightarrow \boldsymbol{\Gamma}_{n}=\mathbf{0}
$$

or

$$
\left(\begin{array}{l}
u^{n_{-}} \\
-\eta^{n_{0}}
\end{array}\right)=\sum_{m \in \mathbb{Z}} \mathbf{B}_{n-m}\left(\begin{array}{l}
u^{n_{0}} \\
\eta^{n_{-}}
\end{array}\right), \quad \mathbf{B}_{-n}=\mathbf{B}_{n}^{*}, \quad|n|>N \rightarrow \mathbf{B}_{n}=\mathbf{0} .
$$

Interaction between the nearest neighbors is introduced by these boundary conditions. We restrict our consideration to the case $N=0, \quad \Gamma_{0}=\left(\begin{array}{ll}0 & \alpha \\ \alpha & 0\end{array}\right)$ :

$$
\left(\begin{array}{l}
u^{n_{-}} \\
\eta^{n_{-}}
\end{array}\right)=\left(\begin{array}{ll}
0 & \alpha \\
\alpha & 0
\end{array}\right) \cdot\left(\begin{array}{l}
u^{n_{0}} \\
\eta^{n_{0}}
\end{array}\right), \quad \Im \mathrm{m} \alpha=0 .
$$

A self adjoint extension $\mathcal{L}(\vec{H}(\tau))$ of the operator $-\Delta_{0} \oplus A_{0}^{\text {int }}$ specified by the boundary conditions (6) simulates the Hamiltonian of the "neutron-lattice" system. Since $\vec{H}=\vec{H}(\tau)$, this Hamiltonian is time-dependent. We consider $\vec{H}(\tau)$ to be a Markovian stochastic process with two stochastic states. The corresponding evolution operator $U(t)$, restricted to a fixed trajectory of the process $\vec{H}(t)$, is the solution of the Cauchy problem:

$$
\frac{1}{i} \cdot \frac{\partial U}{\partial \tau}=\mathcal{L}(\vec{H}(\tau)) U,\left.\quad U\right|_{\tau=0}=I_{q} \equiv I_{e} \oplus I_{i}
$$

where $I_{e}$ and $I_{i}$ are the identity operators in the external and internal spaces respectively.

Together with the stochastic evolution described by equation (7), we will consider the "deterministic" evolutions corresponding to the Hamiltonians $\mathcal{L}(+H)$ and $\mathcal{L}(-H)$, in which the magnetic field is fixed in up-state $\vec{H}=H \vec{e}_{z}$ or in the down state $\vec{H}=-H \vec{e}_{z}$. On the intervals where $\vec{H}(\tau)$ is constant, the evolution equation (7) can be solved by the time-ordered exponentials corresponding to the operators $\mathcal{L}(+H)$ and $\mathcal{L}(-H)$ respectively. On each trajectory of magnetic momentum, the evolution operator (7) is the T-product of the corresponding exponentials (see [6]).

Starting with the equation for the transition probabilities whose resolvent matrix $\mathcal{P}$ represents a solution of the following equation:

$$
\frac{d \mathcal{P}}{d \tau}=\chi\left(\begin{array}{ll}
-1 & 1 \\
1 & -1
\end{array}\right) \mathcal{P}, \quad \mathcal{P}(0)=\left(\begin{array}{ll}
1 & 0 \\
0 & 1
\end{array}\right)
$$

we introduce the measure on the space of trajectories according to the paper [6]. The probability of the beam of trajectories which are in the states $\alpha_{s}= \pm H$ at the moments $t=s \delta, s=0,1,2, \ldots, n$ can be defined by the following formula:

$$
P_{\alpha_{n}, \alpha_{n-1}, \ldots, \alpha_{1}}=\prod_{s=1}^{n}\left\{\exp \left[\chi\left(\begin{array}{ll}
-1 & 1 \\
1 & -1
\end{array}\right)\right] \delta\right\}_{\alpha_{n}, \alpha_{n-1}}
$$


The averaged evolution operator can be calculated by the Trotter formula. By the same method as in [6] the following theorem can be proven:

Theorem 1. The quantum evolution operator, averaged over the set of trajectories of magnetic field starting in the stochastic state $\beta$ at $\tau=0$ and ending in the stochastic state $\alpha$ at $\tau=T$ coincides with the element $\bar{U}_{\alpha \beta}(T)$ of the operator matrix, which satisfies the differential equation

$$
\frac{1}{i} \cdot \frac{\partial}{\partial \tau} \bar{U}=\hat{\mathcal{L}}_{\chi} \bar{U},\left.\quad \bar{U}\right|_{t=0}=\left(\begin{array}{cc}
I_{q} & 0 \\
0 & I_{q}
\end{array}\right) .
$$

Here, the generator $\hat{\mathcal{L}}_{\chi}$ of the averaged semigroup $\bar{U}(\tau)$ is given by the following expression:

$$
\hat{\mathcal{L}}_{\chi}=\left(\begin{array}{ll}
\mathcal{L}(+H) & 0 \\
0 & \mathcal{L}(-H)
\end{array}\right)+i \chi\left(\begin{array}{ll}
I_{q} & 0 \\
0 & I_{q}
\end{array}\right) .
$$

It acts in the quantum-stochastic space $\hat{\mathcal{L}}_{\chi}=\left[L_{2}\left(\mathbb{R}^{3} \oplus E^{\text {int }}\right)\right] \otimes \mathbb{R}^{2}$ which is the tensor product of the quantum space $\mathcal{H}_{q}=L_{2}\left(\mathbb{R}^{3}\right) \oplus E^{\text {int }}$ by the stochastic space $\mathbb{R}^{2}, \mathcal{H}=\mathcal{H}_{q} \oplus \mathcal{H}_{q}$.

\section{Spectral analysis of the averaged operator}

We will consider the perturbed $\hat{\mathcal{L}}_{\chi}$ and the unperturbed operator $\hat{\mathcal{L}}_{\chi}^{0}$ together. The unperturbed operator corresponds to the case when the quantum operator can be presented as the orthogonal sum of the operators in the external and internal spaces. It corresponds to the coupling constant $\alpha$ equal to zero. The external and internal parts of the unperturbed operator are:

$$
\begin{aligned}
-\hat{\Delta} & =\left(\begin{array}{cc}
-\Delta & 0 \\
0 & -\Delta
\end{array}\right)+i \chi\left(\begin{array}{cc}
I_{q} & -I_{q} \\
-I_{q} & I_{q}
\end{array}\right) \\
\hat{A}^{i n t} & =\left(\begin{array}{cc}
A_{i n t}^{u} & 0 \\
0 & A_{i n t}^{d}
\end{array}\right)+i \chi\left(\begin{array}{cc}
I_{i} & -I_{i} \\
-I_{i} & I_{i}
\end{array}\right),
\end{aligned}
$$

where $A_{i n t}^{u, d}=\oplus \sum_{n} A^{u, d} ; A^{u}=A(+H), A^{d}=A(-H)$.

The unperturbed operator $\hat{\mathcal{L}}_{\chi}^{0}=-\hat{\Delta} \oplus \hat{A}^{i n t}$ is normal and its spectral characteristics can be calculated explicitly. For example, the spectrum of this operator is the sum of the spectrum of the operator $-\hat{\Delta}$ (whose spectrum is purely continuous and consists of the two branches: $\lambda=k^{2}$ and $\left.\lambda=k^{2}+2 \imath \chi, \Im \mathrm{m} k=0\right)$ and the spectrum of the operator $\hat{A}^{i n t}$, which consists of four eigenvalues of infinite multiplicity:

$$
\begin{aligned}
& \lambda_{1,2}\left(\hat{A}^{i n t}\right)=\lambda_{0}+i \chi \pm \sqrt{H^{2}-\chi^{2}} \\
& \lambda_{3,4}\left(\hat{A}^{i n t}\right)=\lambda_{1}+i \chi \pm \sqrt{H^{2}-\chi^{2}} .
\end{aligned}
$$

Calculating the resolvent of operator $\hat{\mathcal{L}}_{\chi}$ one can obtain, that the spectrum of $\hat{\mathcal{L}}_{\chi}$ is purely continuous and consists of the following branches:

1) $\mathbb{R}^{+}$and $\mathbb{R}^{+}+2 i \chi$, which coincide with the spectrum of the operator $\hat{\mathcal{L}}_{\chi}^{0}$

2) four branches, or bands, each corresponding to one of the eigenvalues of operator $\hat{\mathcal{L}}_{\chi}^{0}$. These branches can be calculated by solving the following equations:

$$
\lambda-\lambda_{n}\left(\hat{\mathcal{L}}_{\chi}^{0}\right)= \pm \frac{\alpha_{i}^{2}}{32 \pi} \frac{F_{n}(\lambda, t)}{\sqrt{h^{2}-\chi^{2}}}+o\left(\alpha^{2}\right) .
$$

This formula is valid for the small values of the coupling constant $\alpha$ only. Sign "-." in the rhs of (13) corresponds to $n=1,3$, “+ " to $n=2,4$. Function $F_{n}(\lambda, t)$ defined by the following expression:

$$
\begin{gathered}
F_{n}(\lambda, t)=\left(B_{n}^{+} B_{n}^{-}\right)^{-1}\left\{B_{n}^{+}\left(C_{n}^{-} \Delta_{u}^{n}+i \chi_{u}^{n}\right)+B_{n}^{-}\left(C_{n}^{+} \Delta_{d}^{n}+i \chi_{d}^{n}\right)\right\}, \\
\Delta_{u, d}^{n}=R^{+}(\lambda) D_{n}^{ \pm} B_{n}^{\mp}-i \chi R^{-}(\lambda) B_{n}^{ \pm} A_{n}^{\mp}, n=0,1 ; \\
\nabla_{u, d}^{n}=R^{-}(\lambda) D_{n}^{\mp} B_{n}^{ \pm}-i \chi R^{+}(\lambda) B_{n}^{\mp} A_{n}^{ \pm}, n=0,1 ; \\
R^{ \pm}(\lambda)=\hat{B}(\sqrt{\lambda}, t) \pm \hat{B}(\sqrt{\lambda-2 i \chi}, t)
\end{gathered}
$$

where $A_{n}^{ \pm}, B_{n}^{ \pm}, D_{n}^{ \pm}$are defined as in [6]:

$$
A_{0}^{ \pm}=\lambda_{0} \pm H, \quad A_{1}^{ \pm}=\lambda_{1} \mp H,
$$




$$
B_{n}^{ \pm}=A_{n}^{ \pm}-i, \quad D_{n}^{ \pm}=(i \chi-\lambda) A_{n}^{ \pm}-i .
$$

Parameter $t$ in the formulas (13), (14), (16) is the quasimomentum and it belongs to the interval $[-\pi, \pi]$. The function $\hat{B}(\sqrt{\lambda}, t)$ is the lattice sum for the linear infinite chain:

$$
\hat{B}(\sqrt{\lambda}, t)=i k+\sum_{n \in Z} \frac{\exp (i \sqrt{\lambda}|n|)}{4 \pi|n|} \exp (-i t n)=\ln \left(\frac{1}{2\left(\cos \sqrt{-\left(\lambda_{0}+H_{0}\right)}-\cos t\right)}\right),
$$

which was calculated first by Subramanian [10]. The branch of the logarithm is fixed by the condition of analytical continuability of $\hat{B}(\sqrt{\lambda}, t)$ into the complex spectral plane $\lambda$ and vanishing of the imaginary part of the logarithm on the negative semi-axis. The properties of the function $\hat{B}(\sqrt{\lambda}, t)$ were described in [11].

Analysis of the equation (13) can be carried out for the small values of the coupling constant $a \ll 1$ and of the stochastic evolution parameter $\chi \ll 1$. In this case the resonant bands correspond to the negative eigenvalues of the operators $A^{u}=\left(\begin{array}{ll}\lambda_{0}+H & 0 \\ 0 & \lambda_{1}-H\end{array}\right)$ and $A^{d}=\left(\begin{array}{ll}\lambda_{0}-H & 0 \\ 0 & \lambda_{1}+H\end{array}\right)$. For example, let $\lambda_{0}$ be negative. Then the corresponding eigenvalue of the operator $\hat{\mathcal{L}}_{\chi}^{0}$ is given by the following asymptotic expression:

$$
\lambda_{1}\left(\hat{\mathcal{L}}_{\chi}^{0}\right) \lambda_{0}+H+i \chi+o(\chi),
$$

and corresponding band by the expression:

$$
\lambda_{1}(t)=\lambda_{1}\left(\hat{\mathcal{L}}_{\chi}^{0}\right)-\frac{\alpha^{2} i}{8 \pi} H\left[\left(\lambda_{0}+H\right)^{2}+1\right] \ln \frac{1}{2\left(\cos \sqrt{-\left(\lambda_{0}+H\right)}-\cos t\right)}+o\left(\alpha^{2}, \chi\right),
$$

where $\mathrm{ln}$ is defined as a function of the real variable. The right edge of the band coincides with $\lambda(\pi)$ and the left one with $\lambda(0)$. The function $\hat{B}(\sqrt{\lambda}, t)$ is an even function of the variable $t$, hence the multiplicity of the spectrum is two (see Fig. 1).

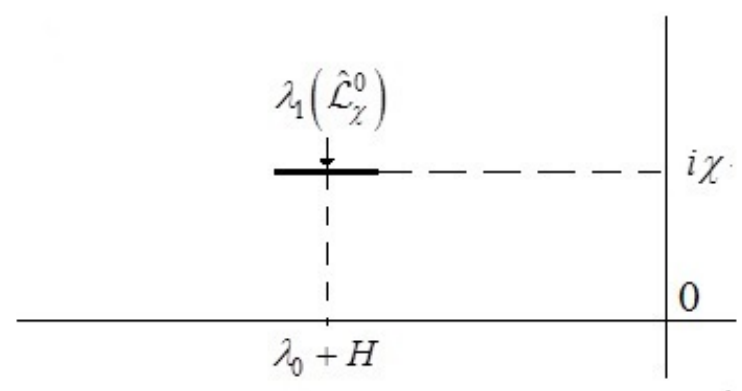

FIG. 1. Spectral band formed by the negative eigenvalue. The right edge of the band coincides with $\lambda_{1}(\pi)$ and the left one with $\lambda_{1}(0)$, where the argument $t$ of the function $\lambda_{1}(t)$ is the quasimomentum (see formula (19) describing the band)

The structure of the band spectrum corresponding to the positive eigenvalues of the operators $A^{u}, A^{d}$ is more complicated. Analysis of the equation (13) shows that the bands corresponding to each positive eigenvalue of $\hat{\mathcal{L}}_{\chi}^{0}$ have a gap (see Fig. 2). Let, for example, $\sqrt{\lambda_{0}+H}$ be from the interval $[0, \pi]$. Then, the band has a gap near $\lambda_{1}\left(\hat{\mathcal{L}}_{\chi}^{0}\right)$. The second band of solutions of the equation (13) is situated exactly under this band, but it does not correspond to the spectrum of the operator. The first band transforms into the stationary (waveguide) band with the gap near $\lambda_{0}+H$ when the parameter $\chi$ tends to zero. The second band transforms into the resonant gap (see Fig. 2 - Spectral band formed by the positive eigenvalue). The first band corresponds to the values of $\lambda$, that are less than $|t|^{2}$, the second - to $\lambda:|\lambda|>|t|^{2}$. When $\sqrt{\lambda_{0}+H}$ is greater than $\pi$, no stationary band appears.

Thus, the spectrum of the operator $\hat{\mathcal{L}}_{\chi}$ consists of two scattered waves branches $\mathbb{R}^{+}$and $\mathrm{R}^{+}+2 i \chi$ and not more than four stationary bands (see Fig. 3). The Bloch waves corresponding to the resonant bands are increasing at infinity functions and are not eigenfunctions of the operator. We are going to prove that the generator $\hat{\mathcal{L}}_{\chi}$ is a dissipative operator with complex branches of the continuous spectrum. The corresponding eigenfunctions can be calculated (see the following papers $[6,11]$ ). The eigenfunctions corresponding to the branches $\mathbb{R}^{+}$and $\mathrm{R}^{+}+2 i \chi$ 


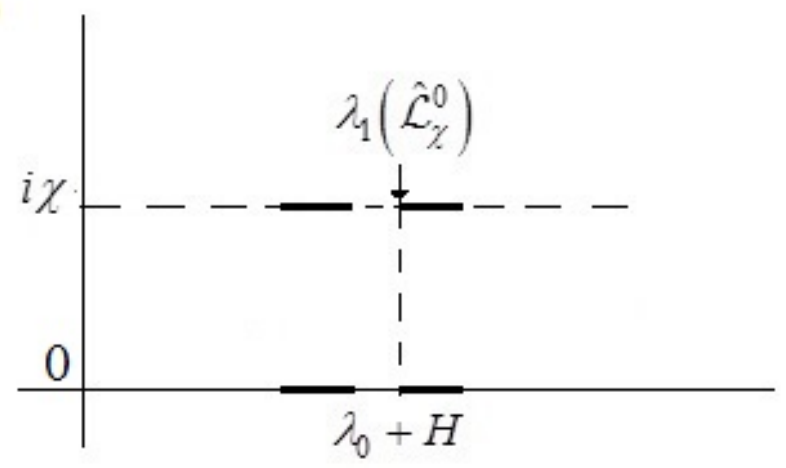

FIG. 2. Spectral band formed by the positive eigenvalue. Analysis of the equation (13) shows that when $\sqrt{\lambda_{0}+H}$ is from the interval $[0, \pi]$, this band have a gap. When $\sqrt{\lambda_{0}+H}$ is greater than $\pi$, no stationary band appears

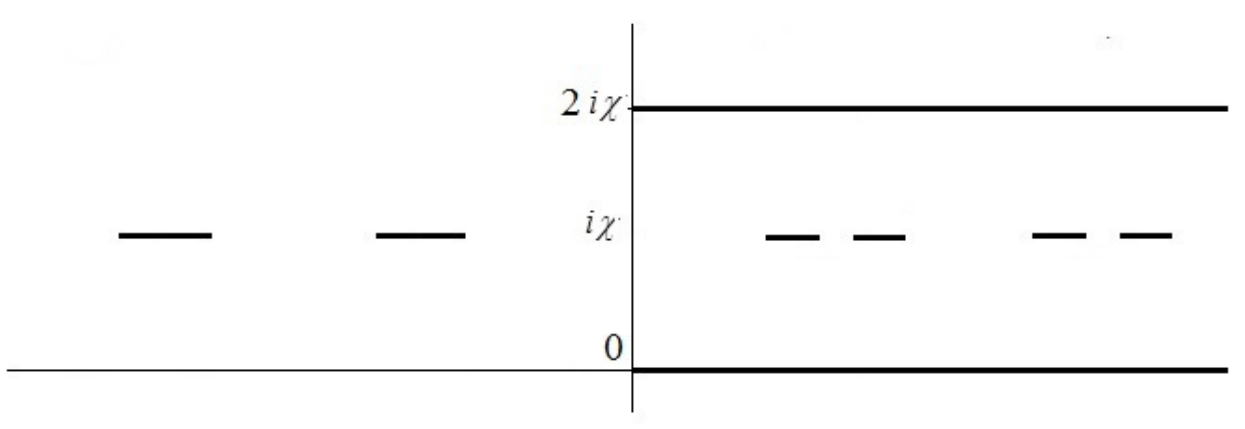

FIG. 3. Spectrum of the averaged operator. It consists of two branches $\mathbb{R}^{+}$and $\mathbb{R}^{+}+2 i \chi$, eigenfunctions corresponding to which have a form of scattered waves, and not more than four stationary bands

have a form of scattered waves. The initial plane wave is symmetric with respect to the stochastic variables for the branch $\mathbb{R}^{+}$(or stable branch) of the spectrum:

$$
\begin{gathered}
\Psi_{s}(\lambda, \boldsymbol{\nu})=\left\{\begin{array}{c}
\psi_{s}^{\text {ext }}(\mathbf{x}, \lambda, \boldsymbol{\nu}) \\
\psi_{s}^{\text {int }}(\lambda, n u)
\end{array}, \lambda=k^{2}, k \geq 0, \boldsymbol{\nu} \in S^{2},\right. \\
\psi_{s}^{\text {ext }}(\mathbf{x}, \lambda, \boldsymbol{\nu})=\exp \{-i \sqrt{\lambda}\langle\boldsymbol{\nu}, \mathbf{x}\rangle\} \cdot\left(\begin{array}{c}
1 \\
1
\end{array}\right)+\left[f_{00}(\lambda, \boldsymbol{\nu}) \sum_{n \in \mathbb{Z}} \frac{\exp \left(i k\left|\mathbf{x}-\mathbf{x}_{n}\right|\right)}{4 \pi\left|\mathbf{x}-\mathbf{x}_{n}\right|}\left(\begin{array}{l}
1 \\
1
\end{array}\right)+\right. \\
\left.+f_{10}(\lambda, \boldsymbol{\nu}) \sum_{n \in \mathbb{Z}} \frac{\exp \left(i \sqrt{\lambda-2 i \chi}\left|\mathbf{x}-\mathbf{x}_{n}\right|\right)}{4 \pi\left|\mathbf{x}-\mathbf{x}_{n}\right|} \cdot\left(\begin{array}{c}
1 \\
-1
\end{array}\right)\right] \exp \left(-i \sqrt{\lambda}\left\langle\boldsymbol{\nu}, \mathbf{x}_{n}\right\rangle\right), \\
\left(\psi_{s}^{\text {int }}(\lambda, \boldsymbol{\nu})\right)_{n}=\left(\begin{array}{c}
\eta_{u}^{0} \\
\eta_{d}^{0}
\end{array}\right)(\lambda, \boldsymbol{\nu}) \exp \left(-i \sqrt{\lambda}\left\langle\boldsymbol{\nu}, \mathbf{x}_{n}\right\rangle\right), \mathbf{x}_{n}=n \mathbf{e} .
\end{gathered}
$$

The initial plane wave corresponding to the relaxation branch $\mathbb{R}^{+}+2 i \chi$ is antisymmetric with respect to the stochastic variables:

$$
\begin{gathered}
\Psi_{a s}(\lambda, \boldsymbol{\nu})=\left\{\begin{array}{c}
\psi_{a s}^{\text {ext }}(\mathbf{x}, \lambda, \boldsymbol{\nu}) \\
\psi_{a s}^{\text {int }}(\lambda, n u)
\end{array}, \lambda=k^{2}+2 i \chi, k \geq 0, \boldsymbol{\nu} \in S^{2}\right. \\
\psi_{\text {as }}^{\text {ext }}(\mathbf{x}, \lambda, \boldsymbol{\nu})=\exp \{-\imath \sqrt{\lambda-2 i \chi}\langle\boldsymbol{\nu}, \mathbf{x}\rangle\} \cdot\left(\begin{array}{c}
1 \\
-1
\end{array}\right)+\left[f_{01}(\lambda, \boldsymbol{\nu}) \sum_{n \in \mathbb{Z}} \frac{\exp \left(i k\left|\mathbf{x}-\mathbf{x}_{n}\right|\right)}{4 \pi\left|\mathbf{x}-\mathbf{x}_{n}\right|} \cdot\left(\begin{array}{c}
1 \\
1
\end{array}\right)+\right.
\end{gathered}
$$




$$
\begin{gathered}
\left.f_{11}(\lambda, \boldsymbol{\nu}) \sum_{n \in \mathbb{Z}} \frac{\exp \left(i \sqrt{\lambda-2 i \chi}\left|\mathbf{x}-\mathbf{x}_{n}\right|\right)}{4 \pi\left|\mathbf{x}-\mathbf{x}_{n}\right|} \cdot\left(\begin{array}{c}
1 \\
-1
\end{array}\right)\right] \exp \left(-i \sqrt{\lambda-2 i \chi}\left\langle\boldsymbol{\nu}, \mathbf{x}_{n}\right\rangle\right), \\
\left(\psi_{\text {as }}^{\text {int }}(\lambda, \boldsymbol{\nu})\right)_{n}=\left(\begin{array}{c}
\eta_{u}^{0} \\
\eta_{d}^{0}
\end{array}\right)(\lambda, \boldsymbol{\nu}) \exp \left(-i \sqrt{\lambda-2 i \chi}\left\langle\boldsymbol{\nu}, \mathbf{x}_{n}\right\rangle\right), \mathbf{x}_{n}=n \mathbf{e} .
\end{gathered}
$$

One can see, that the functions $\psi_{s}^{e x t}, \psi_{a s}^{e x t}$ satisfy Bloch conditions. For example:

$$
\psi_{s}^{e x t}(\mathbf{x}+m \tilde{\mathbf{e}}, \lambda, \boldsymbol{\nu})=\psi_{s}^{e x t}(\mathbf{x}, \lambda, n u) \cdot \exp (-i \sqrt{\lambda}\langle\boldsymbol{\nu}, m \overrightarrow{\mathbf{e}}\rangle), \quad m \in \mathbb{Z}
$$

Explicit expressions for the amplitudes $f_{m n}(\lambda, \nu)$ in (21), (23) can be calculated by substitution of the considering ansatz (21), (23) for the scattered waves into the boundary conditions (6). For example, the amplitude $f_{00}(\lambda, \boldsymbol{\nu})$ is:

$$
f_{00}(\lambda, \boldsymbol{\nu})=\frac{\alpha^{2}}{4} \sum_{n, m=0}^{1} \frac{\left(\lambda_{n}-(-1)^{n+m} H-\lambda\right)\left(\lambda_{n}+(-1)^{n+m} H-i\right)+2 \imath \chi\left(\lambda_{n}-i\right)}{\left[\left(\lambda_{n}-i\right)^{2}-H^{2}\right]\left[\left(\lambda_{n}+i \chi-\lambda\right)^{2}-\left(H^{2}-\chi^{2}-F_{k}\left(\sqrt{\lambda}, k_{\|}\right)\right)\right]} Z_{n}^{m}+o\left(\alpha^{2}\right),
$$

where the following notations were used:

$$
k_{\|}=k\langle\boldsymbol{\nu}, \mathbf{e}\rangle ; \quad k=\sqrt{\lambda} ; \quad Z_{m}^{0}=Z_{m}^{+}, Z_{m}^{1}=Z_{m}^{-} ; \quad Z_{m}^{ \pm}=i \chi B_{m}^{ \pm} A_{m}^{ \pm}-D_{m}^{ \pm} B_{m}^{ \pm}
$$

The eigenfunctions corresponding to the stationary band can be calculated in the same way

$$
\Psi_{j}(t)=\left\{\begin{array}{c}
\psi_{j}^{\text {ext }}(\mathbf{x}, t) \\
\psi_{j}^{\text {int }}(t)
\end{array}\right.
$$

Let us denote by $\lambda_{j}(t)$ the corresponding solution of the equation (13). Then the components of the eigenfunction are:

$$
\begin{gathered}
\psi_{j}^{e x t}(\mathbf{x}, t)=\frac{1}{2} \sum_{n \in \mathbb{Z}}\left[C_{0} \frac{\exp \left(i \cdot \sqrt{\lambda_{j}(t)}\left|\mathbf{x}-\mathbf{x}_{n}\right|\right)}{4 \pi\left|\mathbf{x}-\mathbf{x}_{n}\right|}\left(\begin{array}{c}
1 \\
1
\end{array}\right)+\right. \\
\left.+C_{\chi} \frac{\exp \left(i \cdot \sqrt{\lambda_{j}(t)-2 i \chi}\left|\mathbf{x}-\mathbf{x}_{n}\right|\right)}{4 \pi\left|\mathbf{x}-\mathbf{x}_{n}\right|}\left(\begin{array}{c}
1 \\
-1
\end{array}\right)\right] \cdot \exp (-i n t), \\
\psi_{j}^{i n t}(t)=\left(\begin{array}{c}
\eta_{u}^{0} \\
\eta_{d}^{0}
\end{array}\right)(j, t) \exp (-i n t)
\end{gathered}
$$

where the following notations were used:

$$
\begin{gathered}
C_{0, \chi}=\text { const } \cdot \alpha\left(C_{m}^{-} B_{m}^{+} \pm i \chi B_{m}^{-}\right)+o\left(\alpha^{2}\right) ; \\
m=0 \text { for } j=1,2 \text { and } m=1 \text { for } j=3,4 ; \\
C_{m}^{ \pm}\left(\lambda_{j}(t)\right)=A_{m}^{ \pm}+i \chi-\lambda_{j}(t) .
\end{gathered}
$$

\section{Eigenfunction-expansion theorem}

We will restrict our consideration to the case of the initial data with the trivial "internal" component, i.e. we will consider functions:

$$
\hat{f}\left(\begin{array}{ll}
f_{u} & \xi_{u} \\
f_{d} & \xi_{d}
\end{array}\right), \quad \xi_{u}=\xi_{d}=0
$$

The external part of the function can be defined as follows:

$$
[\hat{f}]^{e x t}(\mathbf{x})=\left(\begin{array}{c}
f_{u} \\
f_{d}
\end{array}\right)(\mathbf{x}) \equiv f(\mathbf{x}), \quad f_{u}, f_{d} \in L_{2}\left(\mathbb{R}^{3}\right) .
$$

The following assertion can be proven:

Theorem 2. Let the vector $\hat{f}$ from the quantum-stochastic space $\mathcal{H}$ has the form (30), then the following representation holds almost everywhere in the Lebesque measure sense:

$$
f(\mathbf{x})=\sum_{j=1}^{4} K_{i} \int_{\Delta_{j}} d t \int_{\mathbb{R}^{3}} d \mathbf{y} \psi_{j}^{e x t}(\mathbf{x}, t) \overline{\phi_{j}^{e x t}(\mathbf{y}, t)} f(\mathbf{y})+
$$




$$
+\frac{1}{16 \pi^{3}} \int_{\mathbb{R}^{+}} k^{2} d k \int_{S^{2}} d \boldsymbol{\nu} \int_{\mathrm{R}^{3}} d \mathbf{y}\left\{\psi_{s}^{e x t}(\mathbf{x}, k, \boldsymbol{\nu}) \overline{\phi_{s}^{e x t}(\mathbf{y}, t)}+\psi_{a s}^{e x t}(\mathbf{x}, k, \boldsymbol{\nu}) \overline{\phi_{a s}^{e x t}(\mathbf{y}, t)}\right\} f(\mathbf{y}) .
$$

Vector-valued functions $\phi_{i}(\mathbf{y}, t), \phi_{s, a s}(\mathbf{y}, k, \nu)$ are externals parts of the eigenfunctions of the adjoint operator $\hat{\mathcal{L}}_{\chi}^{*}$ corresponding to the stationary bands and branches of the continuous spectrum $\mathbb{R}^{+}$and $\mathbb{R}^{+}+2 i \chi$ respectively. The intervals $\Delta_{j}=\left[-\alpha_{j},-\beta_{j}\right] \cup\left[\beta_{j}, \alpha_{j}\right]$ are introduced in such a way, that the spectral parameter $\lambda_{j}(t)$ covers the stationary band twice, when the quasimomentum varies on the interval $\Delta_{j}$.

This theorem can be proved by integrating by parts the bilinear form of the resolvent of the operator $\hat{\mathcal{L}}_{\chi}$ around the spectrum. This theorem allows us to calculate the averaged evolution operator $\bar{U}$, which will be used to derive the scattering operator:

$$
\begin{gathered}
{[\bar{U}(\tau) \hat{f}]^{e x t}(x)=\left[\exp i \hat{\mathcal{L}}_{\chi} \tau \hat{f}^{e x t}\right](\mathbf{x})=} \\
\sum_{j=1}^{4} K_{j} \int_{\Delta_{j}} d t \cdot \exp \left(i \lambda_{j}(t) \tau\right) \int_{\mathbb{R}^{3}} d \mathbf{y} \cdot \psi_{j}^{\text {ext }}(\mathbf{x}, t) \overline{\phi_{j}^{e x t}(\mathbf{y}, t)} f(\mathbf{y})+ \\
+\frac{1}{16 \pi^{3}} \int_{\mathbb{R}^{+}} \exp \left(i k^{2} \tau\right) k^{2} d k \iint_{S^{2}} d \boldsymbol{\nu} \int_{\mathbb{R}^{3}} d \mathbf{y} \cdot \psi_{s}^{\text {ext }}(\mathbf{x}, k, \boldsymbol{\nu}) \overline{\phi_{s}^{e x t}(\mathbf{y}, t)} f(\mathbf{y})+ \\
+\frac{1}{16 \pi^{3}} \int_{\mathbb{R}^{+}} \exp \left(i\left(k^{2}+2 i \chi\right) \tau\right) k^{2} d k \int_{S^{2}} d \boldsymbol{\nu} \int_{\mathbb{R}^{3}} d \mathbf{y} \cdot \psi_{a s}^{\text {ext }}(\mathbf{x}, k, \boldsymbol{\nu}) \overline{\phi_{a s}^{\text {ext }}(\mathbf{y}, t)} f(\mathbf{y})
\end{gathered}
$$

\section{Scattering operator}

The averaging of the quantum evolution leads to the evolution operator semigroup with the generator $\hat{\mathcal{L}}_{\chi}$ :

$$
\bar{U}(\tau)=\exp \left(i \hat{\mathcal{L}}_{\chi} \tau\right), \quad \tau>0
$$

which acts in the quantum-stochastic space $\mathcal{H}$. Formula (33) shows that the contribution of the nonreal part of the spectrum tends to zero for large $\tau \rightarrow \infty$. As a result, only the real branch of spectrum $\mathbb{R}^{+}$contributes to the scattering process. We will define the unperturbed operator for the scattering problem as the restriction of the operator $-\hat{\Delta}$ to the stable invariant subspace corresponding to the real branch of the continuous spectrum $\mathbb{R}^{+}$. The corresponding operator will be denoted by $\hat{L}_{0}$. It is unitarily equivalent to the nonperturbed Laplacian defined on the domain $W_{2}^{2}\left(\mathbb{R}^{3}\right)$. The identification operator $J=J_{0}$ is the projector on the set $H_{s}$ of the functions which are symmetric with respect to the stochastic variables. In this way we eliminate the relaxation branch and the scattering matrix can be defined as follows:

$$
\bar{S}_{\chi}\left(\alpha, \hat{L}_{0}\right)=s-\lim _{\tau \rightarrow \infty} \exp \left(-i \hat{L}_{0} \tau\right) J_{0} \exp \left(2 i \hat{\mathcal{L}_{\chi}} \tau\right) J_{0}^{*} \exp \left(-i \hat{L}_{0} \tau\right)
$$

Using the unitary operator $\Sigma: H_{s} \rightarrow L_{2}\left(\mathbb{R}^{3}\right)$ :

$$
\Sigma: \hat{f}=\left(\begin{array}{c}
f \\
f
\end{array}\right) \rightarrow \frac{1}{2}(f+f)=f
$$

the averaged scattering operator from $L_{2}\left(\mathbb{R}^{3}\right)$ to $L_{2}\left(\mathbb{R}^{3}\right)$ can be written in the following form:

$$
\bar{S}_{\chi}(\alpha)=s-\lim _{\tau \rightarrow \infty} \exp (i \Delta \tau) \Sigma J_{0} \bar{U}(2 \tau) J_{0}^{*} \Sigma^{*} \exp (i \Delta \tau)
$$

Then the averaged scattering matrix can be calculated:

$$
\bar{S}_{\chi}\left(\mathbf{p}, \mathbf{p}^{\prime}\right)=\delta\left(\mathbf{p}-\mathbf{p}^{\prime}\right)-\frac{1}{4 \pi^{2}} \delta\left(\mathbf{p}^{2}-\left(\mathbf{p}^{\prime}\right)^{\mathbf{2}}\right) \cdot f_{00}\left(|\mathbf{p}|,-\frac{\mathbf{p}}{|\mathbf{p}|}\right) \sum_{n \in \mathbb{Z}} \delta\left(2 \pi n+\left\langle\mathbf{p}-\mathbf{p}^{\prime}, \mathbf{e}\right\rangle\right),
$$

Thus the scattering amplitude $f(\boldsymbol{\omega}, \boldsymbol{\nu}, k)$ has the following form:

$$
f(\boldsymbol{\omega}, \boldsymbol{\nu}, k)=-f_{00}(k, \boldsymbol{\nu}) \cdot \sum_{n \in \mathbb{Z}} \delta(2 \pi n+k\langle\boldsymbol{\nu}+\boldsymbol{\omega}, \mathbf{e}\rangle),
$$

where $f_{00}(k, \nu)$ depends on the direction of the initial plane wave trough the projection of the vector $k \boldsymbol{\nu}$ on the lattice vector $\tilde{\mathbf{e}}$ :

The scattering amplitude has Laue singularities.

$$
k_{\|}=k\langle\boldsymbol{\nu}, \mathbf{e}\rangle \text {. }
$$


It is important to discuss the limit of the scattering amplitude when the stochastic parameter tends to zero. We will denote by $f^{ \pm}(\boldsymbol{\omega}, \boldsymbol{\nu}, k)$ the scattering amplitudes corresponding to the operators with the fixed stochastic states: $\mathcal{L}(+H)$ and $\mathcal{L}(-H)$. Then the following formula can be derived:

$$
\lim _{\chi \rightarrow 0} f(\boldsymbol{\omega}, \boldsymbol{\nu}, k)=\frac{1}{2}\left[f^{+}(\boldsymbol{\omega}, \boldsymbol{\nu}, k)+f^{-}(\boldsymbol{\omega}, \boldsymbol{\nu}, k)\right] .
$$

This formula shows that the limit amplitude is equal to the arithmetic average of the amplitudes corresponding to the deterministic processes.

\section{Acknowledgement}

This work was initiated by my professor B.S. Pavlov, an outstanding mathematician of the St. Petersburg mathematical school who passed away more than two years ago.

The author wants to thank professor P. B. Kurasov for the scientific support in solving the problem under consideration.

\section{References}

[1] Berezin F.A., Faddeev L.D. Remark on the Schrödinger equation with singular potential. Dokl. Akad. Nauk SSSR, 1961, 137(5), P. 10111014 (in Russian).

[2] Pavlov B.S. Model of a zero-range potential with internal structure. Theoret. and Math. Phys., 1984, 59(3), P. 544-550.

[3] Pavlov B.S., The theory of extensions and explicitly-soluble models. Russian Math. Surveys, 1987, 42(6), P. 127-168.

[4] Albeverio S. and Kurasov P. Singular perturbations of differential operators. Solvable Schrodinger type operators. London Mathematical Society Lecture Notes 271, Cambridge Univ. Press, Cambridge 2000, 444 p.

[5] Boitsev A.A., Neidhardt H., Popov I.Y. Dirac operator coupled to bosons. Nanosystems: Physics, Chemistry, Mathematics, 2016, 7(2), P. 332-339.

[6] Pavlov B.S., Ryzhkov A.E. Scattering on a random point potential, in P.Exner, P.Seba Applications of Self-Adjoint Extensions in Quantum Physics, Lecture Notes in Physics no 324, Springer-Verlag, Berlin-Heidelberg-New York, 1989, P. 100-114.

[7] Ryzhkov A.E. Scattering of the acoustic waves on a Markovian point defect, in P. Exner, P. Seba. Schroedinger Operators, Standard and Nonstandard, World Scientific, Singapore, 1989, P. 407-409.

[8] Albeverio S., Gesztesy F., Hoegh-Krohn R. and Holden H., with an appendix by P. Exner. Solvable Models in Quantum Mechanics, 2-nd ed., American Mathematical Society, Providence, Rhode Island, 2005, 488 p.

[9] Karpeshina Yu.E. Eigenfunction-expansion theorem of scattering problems on one-dimensional periodic supports of the chain type in three-dimensional space. Problemy Mat. Fiz., 1983, 10, P. 137-163 (in Russian).

[10] Subramanian R. Some applications of the method of zero-range potentials in quantum mechanics. Author's Abstract of Candidate's Dissertation, Leningrad State University, 1986 (in Russian).

[11] Kurasov P.B., Pavlov B.S. Electron in a homogeneous crystal of point atoms with internal structure. II. Theoret. and Math. Phys., 1988, 74(1), P. 58-66. 